\title{
Intramolecular dynamics by photoelectron spectroscopy. II. Nonadiabatic processes
}

\author{
D. Dehareng, B. Leyh,, M. Desouter-Lecomte, J. C. Lorquet, J. Delwiche, ${ }^{\text {b) }}$ and M. J. Hubin- \\ Franskin ${ }^{\text {b) }}$
}

Département de Chimie, Université de Liège, Sart-Tilman, B-4000 Liège 1, Belgium

(Received 7 June 1983; accepted 13 July 1983)

\begin{abstract}
The Fourier transform of an electronic spectrum leads to an autocorrelation function which provides information on the propagation of a wave packet on the potential energy surface of the electronic state reached in the transition. The formula is valid even when nonadiabatic interaction is present, i.e., when the wave packet splits at a particular surface crossing with one part branching off to another potential energy surface. An explicit expression of the correlation function is given for a model of several discrete states interacting with a continuum. Closed-form solutions are given in the case of one and two resonances. A very simple formula valid in the strong coupling limit is also derived. The method is applied to the photoelectron spectrum of state $A^{2} \Sigma^{+}$of $\mathrm{HBr}^{+}$, which is shown to correspond to the strong coupling case.
\end{abstract}

\section{INTRODUCTION}

In 1978, Heller demonstrated that an electronic spectrum could be converted into an autocorrelation function by a Fourier transform operation. ${ }^{1}$ Such a correlation function describes the evolution in time of the wave packet created by the Franck-Condon transition, as it propagates on the potential energy surface of the electronic upper state. More precisely, it is equal to the modulus of the overlap integral between the initial position of the wave packet and its instantaneous position at some later time $t$. This technique thus allows a visualization of wave packet motion on a potential energy surface and has proven its usefulness in problems of intramolecular dynamics. ${ }^{2,3}$ In practice, the method is limited to a time range which does not exceed $10^{-13} \mathrm{~s}$ after the initial excitation. Therefore, only very fast molecular processes taking place within the first few molecular vibrational periods can be studied.

It is well known that nonadiabatic processes often give rise to such ultrafast processes, ${ }^{4-?}$ First, internal conversions are among the fastest processes known. ${ }^{4} \mathrm{Re}$ cent research has shown that they were still faster when brought about by a conical intersection. ${ }^{5-7}$ Correlation functions pertaining to such situations have been obtained and analyzed by Fourier transformation of a calculated spectrum. ${ }^{7}$

The present paper is organized as follows. In Sec. II, we demonstrate that the validity of Heller's formula extends to cases for which discrete states interact with a dissociation continuum. We then work out (Sec. III) an analytic expression for the correlation function which reduces to a very simple expression in the case of strong coupling, i. e., in the case of very fast predissociations. In Sec. IV, the predissociation of the $A^{2} \Sigma^{+}$state of $\mathrm{HBr}^{*}$ by a repulsive quartet is analyzed and is shown to correspond to this limit case.

\footnotetext{
a) Boursier de l'Institut pour l'Encouragement de la Recherche Scientifique dans l'Industrie et l'Agriculture, Belgium.

${ }^{b)}$ Chercheur Qualifié du Fonds National de la Recherche Sclentifique, Belgium.
}

\section{NONADIABATIC PROCESSES}

A demonstration of the validity of Heller's formula is implicitly contained in Mies and Krauss's paper, ${ }^{8}$ but this result was not explicitly stated by these authors. Let us consider a situation (represented in Fig. 1) in which a series of zero-order discrete states $\left|\varphi_{j}\right\rangle$ inter act with a dissociation continuum $|E\rangle$. In the corresponding predissociation problem, all the levels which are above the dissociation asymptote are in principle coupled with the continuum, but the interaction becomes large only at the crossing point, or slightly below it (because of the tunnel effect). In a model, it is sensible to introduce a certain critical energy $E_{c}$ above which all the upper members of set $|\varphi\rangle$ interact with the continuum by a coupling operator $V$ which may result either from spinorbit interaction, or from nonadiabatic coupling associated with the breakdown of the Born-Oppenheimer approximation. Direct optical transition to the continuum states is assumed to be forbidden. The populated states are the members of the discrete set $\left|\varphi_{j}\right\rangle$. Since they are not eigenstates of the total Hamiltonian, evolution in time takes place. As a result, dissociation proceeds.

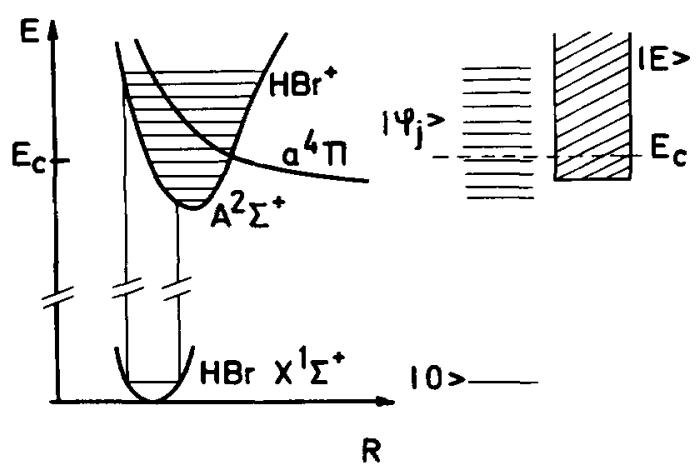

FIG. 1. Coupling between discrete states and a dissociation continuum. The discrete states $\left|\varphi_{j}\right\rangle$ whose energy is above the dissociation asymptote are in principle coupled with the continuum, but the coupling becomes noticeable only when the energy is greater than a certain limit $E_{c}$. 
What is the meaning of the Fourier transform of the optical profile under such conditions? Below energy $E_{c}$, the spectrum is discrete and the eigenfunctions are given by a set $\left.\left\{\mid \varphi_{k}\right)\right\}\left(0 \leq k \leq n-1 ; E_{k}<E_{c}\right)$. Above $E_{c}$ the spectrum is continuous; following the Fano-Mies-Krauss theory, ${ }^{8,9}$ we expand the eigenfunctions $\left|\Psi_{E}\right\rangle$ over the zero-order functions:

$$
\left|\Psi_{E}\right\rangle=\sum_{j=n}^{N} a_{j}\left|\varphi_{j}\right\rangle+\int_{E_{c}}^{+\infty} d E^{\prime} b_{E^{\prime}}(E)\left|E^{\prime}\right\rangle,
$$

where $\left|\varphi_{n}\right\rangle$ is the first state coupled to the continuum.

We adopt the usual assumptions concerning coupling matrix elements;

$$
\begin{aligned}
& \left\langle\varphi_{i}|V| \varphi_{j}\right\rangle=0, \\
& \left\langle E^{\prime}|V| E\right\rangle=0, \\
& \left\langle\varphi_{j}|V| E\right\rangle=V=\text { Cte } \quad(n \leq j \leq N), \\
& \Gamma=\pi|V|^{2} .
\end{aligned}
$$

Thus, in the absence of any better information, we resort to the usual assumption of a coupling matrix element which is independent of energy and which has the same value for all resonances. We assume further that only discrete states $\left|\varphi_{j}\right\rangle$ can be populated from the electronic ground state $|0\rangle$. Thus, at time $t=0$, the expres sion of the wave packet is given by ${ }^{8}$

$$
|\phi(0)\rangle=\sum_{j=0}^{N} T_{0 j}\left|\varphi_{j}\right\rangle
$$

with

$$
T_{0 j}=\left\langle\varphi_{j}|T| 0\right\rangle,
$$

whereas

$$
\left\langle E^{\prime}|T| 0\right\rangle=0 \text {, }
$$

where $T$ is the dipole-moment operator.

The spectral intensity is then given by

$$
I(E) / E=\sum_{j=0}^{n-1}\left|T_{0 j}\right|^{2} \delta\left(E-E_{j}\right), \quad \text { when } E<E_{c}
$$

and

$$
I(E) / E=\left|\sum_{j=n}^{N} a_{j}(E)^{*} T_{0 j}\right|^{2}, \quad \text { when } E>E_{c} .
$$

Following Mies and Krauss, the expression of the propagated wave packet is given by

$$
\begin{aligned}
|\phi(t)\rangle= & \sum_{k=0}^{n-1} T_{0 k} \exp \left(-i E_{k} t / \hbar\right)\left|\varphi_{k}\right\rangle \\
& +\sum_{k=n}^{N} \int_{E_{C}}^{+\infty} d E \exp (-i E t / \hbar) a_{k}(E)^{*} T_{0 k}\left|\Psi_{E}\right\rangle .
\end{aligned}
$$

This first term constitutes the contribution of the discrete eigenstates $\left(E<E_{c}\right)$, whereas the second one implies the continuum states $\left(E>E_{c}\right)$. Multiplying Eq. (II. 3) and (II. 7), we obtain easily:

$$
\begin{aligned}
& \langle\phi(0) \mid \phi(t)\rangle=\sum_{j=0}^{n=1}\left|T_{0 j}\right|^{2} \exp \left(-i E_{j} t / \hbar\right) \\
& +\sum_{j=n}^{N} \sum_{k=n}^{N} T_{j j}^{*} T_{0 k} \int_{E_{c}}^{+\infty} d E a_{j}(E) a_{k}(E)^{*} \exp (-i E t / \hbar)
\end{aligned}
$$

$$
\begin{aligned}
\langle\phi(0) \mid \phi(t)\rangle= & \int_{-\infty}^{E_{c}} d E\left[\sum_{j=0}^{n-1}\left|T_{0 j}\right|^{2} \delta\left(E-E_{j}\right)\right] \exp (-i E t / \hbar) \\
& +\int_{E_{c}}^{+\infty} d E\left|\sum_{j=n}^{N} T_{0 j} a_{j}(E)\right|^{2} \exp (-i E t / \hbar) \text {. (II. 8) }
\end{aligned}
$$

Comparing with Eq. (II. 6), we conclude that

$$
\langle\phi(0) \mid \phi(t)\rangle=\int_{-\infty}^{+\infty} \frac{I(E)}{E} \exp (-i E t / \hbar) d E .
$$

This establishes the validity of Heller's formula in a situation where discrete states interact with a continuum. It is thus possible to study by this method electronic relaxation processes brought about by nonadiabatic or spinorbit coupling among potential energy surfaces. A first example has been recently studied by Köppel. ?

\section{EXPRESSION OF THE CORRELATION FUNCTION}

We now attempt to derive an explicit expression for the correlation function. Two methods are a priori possible to calculate the time-dependent wave function $|\phi(t)\rangle$. They differ in the nature of the basis set used in the expansion. In the first method, ${ }^{10}$ the propagated wave packet is expanded in the set of eigenfunctions of the zero-order Hamiltonian $H_{0}$ :

$$
\begin{aligned}
|\phi(t)\rangle= & \sum_{j=0}^{n-1} b_{j}(0) \exp \left(-i E_{j} t / \hbar\right)\left|\varphi_{j}\right\rangle \\
& +\sum_{j=n}^{N} b_{j}(t) \exp \left(-i E_{j} t / \hbar\right)\left|\varphi_{j}\right\rangle \\
& +\int_{E_{c}}^{+\infty} d E b(E, t) \exp (-i E t / \hbar)|E\rangle .
\end{aligned}
$$

When the simplifying assumptions (II. 2) are made, this method leads to a set of coupled differential equations with a very simple form

$$
\dot{b}_{k}(t)=-\frac{\Gamma}{\hbar} \sum_{j=n}^{N} b_{j}(t) \exp \left[i\left(E_{k}-E_{j}\right) t / \hbar\right]
$$

with $k=n, \ldots, N$.

These equations can be numerically integrated (e.g. , by the Adams-Moulton method ${ }^{11}$ ). Equation (III. 1) can be converted into a correlation function which clearly exhibits the contribution of bound and coupled levels:

$$
\begin{aligned}
C(t)= & |\langle\phi(0) \mid \phi(t)\rangle|=\left.\left|\sum_{j=0}^{n-1}\right| b_{j}(0)\right|^{2} \exp \left(-i E_{j} t / \hbar\right) \\
& +\sum_{j=n}^{N} b_{j}(0)^{*} b_{j}(t) \exp \left(-i E_{j} t / \hbar\right) \mid .
\end{aligned}
$$

Alternatively, a Fano-Mies-Krauss ${ }^{8.9}$ expansion in the set of eigenfunctions $\left|\Psi_{E}\right\rangle$ of the total Hamiltonian $H_{T}$ can be carried out [Eqs. (П. 3), (II. 7), and (II. 8)]. The correlation function then takes the form

$$
\begin{aligned}
C(t)= & |\langle\phi(0) \mid \phi(t)\rangle|=\left.\left|\sum_{j=0}^{n+1}\right| T_{0 j}\right|^{2} \exp \left(-i E_{j} t / \hbar\right) \\
& +\sum_{j=n}^{N} \sum_{k=n}^{N} T_{0 j}^{*} T_{0 k} I_{k j}(t) \mid
\end{aligned}
$$

where the matrix elements $I_{k j}$ are equal to

$I_{k j}(t)=\int_{E_{C}}^{+\infty} d E a_{k}(E)^{*} a_{j}(E) \exp (-i E t / \hbar)$. 
Since $a_{k}^{*}(E) a_{j}(E)$ decreases rapidly as the energy goes below $E_{c}, I_{k f}$ can be calculated by replacing $E_{c}$ by $-\infty$. We can then use the theorem of residues with a semicircular infinite contour in the lower half plane. A few additional details on both methods are given in the Appendix.

Three terms appear in the general expressions of the square of the correlation function as given by Eqs. (III. 3) or (III. 4):

$$
C(t)^{2}=F_{1}(t)+F_{2}(t)+F_{3}(t),
$$

$F_{1}$ is associated with the stable levels $\left|\varphi_{1}\right\rangle$; it always has the following expression:

$$
F_{1}(t)=\sum_{j=0}^{n+1} \sum_{k=0}^{n e d} T_{j} T_{k} \cos \left[\left(E_{j}-E_{k}\right) t / \hbar\right]
$$

with

$$
T_{j}=\left|b_{j}(0)\right|^{2}=\left|T_{0 j}\right|^{2} \text {. }
$$

$T_{j}$ and $T_{k}$ are thus Franck-Condon factors. $F_{2}$ is associated with the resonances and $F_{3}$ is the interference term. When a single level only $\left|\varphi_{\alpha}\right\rangle$ is coupled to the continuum, one has

$$
\begin{aligned}
& F_{2}=T_{\alpha}^{2} \exp (-2 \Gamma t / \hbar), \\
& F_{3}=2 \sum_{j=0}^{n-1} T_{j} T_{\alpha} \exp (-r t / \hbar) \cos \left[\left(E_{j}-E_{\alpha}\right) t / \hbar\right] .
\end{aligned}
$$

Thus, the contribution of the resonance decreases exponentially both with time and with the magnitude of the coupling parameter.

When two states $\left|\varphi_{\alpha}\right\rangle$ and $\left|\varphi_{\beta}\right\rangle$ are coupled with the continuum, an analytical solution is also possible. The solution involves the Mies-Krauss parameter ${ }^{8}$

$$
R=\Gamma /\left|E_{\theta}-E_{\alpha}\right|=\Gamma / \Delta E ;
$$

when $R<0.5$, two related quantities appear:

$$
\begin{aligned}
& \delta=\left(1-4 R^{2}\right)^{1 / 2}, \\
& \phi=\Delta E \delta / 2 \hbar .
\end{aligned}
$$

One finds that $F_{2}$ and $F_{3}$ contain the same exponentially decreasing factors modulated by oscillatory functions:

$$
\begin{aligned}
F_{2}= & \exp (-2 \Gamma t / \hbar)\left\{\left(T_{\alpha}+T_{\beta}\right)^{2} \cos ^{2} \phi t\right. \\
& +\delta^{-2}\left[\left(T_{\alpha}-T_{\beta}\right)^{2}+16 T_{\alpha} T_{\beta} R^{2}\right] \sin ^{2} \phi t \\
& \left.-8 R \delta^{-2}\left(T_{\alpha} T_{\beta}\right)^{1 / 2}\left(T_{\alpha}+T_{\beta}\right) \cos \phi t \sin \phi t\right\},
\end{aligned}
$$

$$
\begin{aligned}
F_{3}= & 4 \exp (-\Gamma t / \hbar)\left\{\sum_{j=0}^{n-1} T_{j} \cos \left[\left(E_{1}+E_{2}\right) / 2-E_{j}\right] t / \hbar\right. \\
& \left.\times\left[\left(T_{\alpha}+T_{\beta}\right) \cos \phi t-(R / \delta)\left(T_{\alpha} T_{B}\right)^{1 / 2} \sin \phi t\right]\right\} .
\end{aligned}
$$

When $R>0.5$, the solution has the same form, except that parameter $\delta$ is replaced by a quantity $\gamma$ equal to $\left(4 R^{2}-1\right)^{1 / 2} ; \phi$ is then equal to $\Delta E \gamma / 2 \hbar$ and the circular functions $\sin$ and $\cos$ must be replaced by hyperbolic functions sinh and cosh.

The contribution of $F_{2}$ and $F_{3}$ may again be expected to become negligible after a sufficient lapse of time if the coupling is large enough. The correlation function then reduces to $\sqrt{F_{1}}$ only, i. e., to a very simple expression

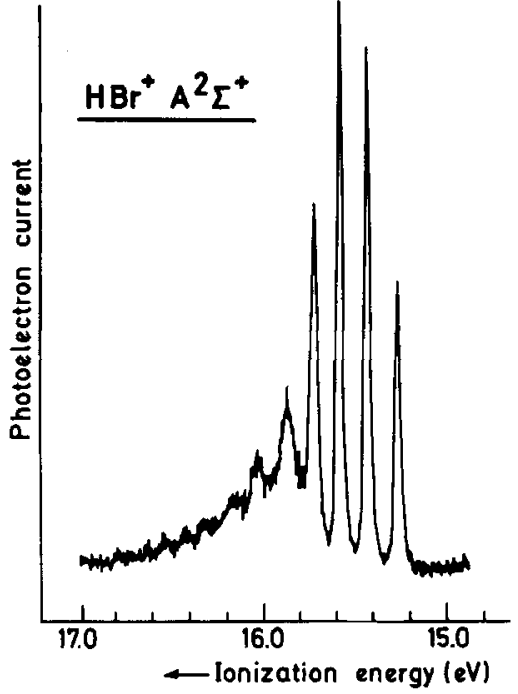

FIG. 2. HeI (58.4 nm) photoelectron spectrum of the $A^{2} \Sigma^{+}$ionic state of $\mathrm{HBr}$.

whose evaluation is immediate once the energies $E$, and Franck-Condon factors $T$, of the unpredissociated levels are known.

When more than two levels are coupled with the continuum, an analytic expression of $F_{2}$ and $F_{3}$ cannot be derived, and the solution has to be calculated numerically. This has been done for various coupling strengths $\Gamma$ and for a number of predissociated levels equal to six. It was observed that, the larger the number of resonances, the smaller the contribution of $F_{2}$ and $F_{3}$ and the sooner $\sqrt{F_{1}}$ becomes equal to the genuine correlation function.

The physical significance of a situation in which $C(t)$ is practically equal to $\sqrt{F_{1}}(t)$ already at the first resonance is then clear. The wave packet is initially prepared in the Franck-Condon zone. After it has gone through the nonadiabatic coupling region (i. e., after half a vibrational period), it splits into two parts. The first part, which results from the superposition of low-energy vibrational bound states, remains trapped in the attractive potential. The second part of the wave packet is made up of high-energy components coupled with the continuum. After undergoing a nonadiabatic transition to the repulsive state, it travels away along the dissociation asymptote.

\section{PREDISSOCIATION OF STATE $A^{2} \Sigma^{+}$OF $\mathrm{HBr}^{+}$}

The photoelectron spectrum of state $A^{2} \Sigma^{+}$exhibits a sequence of four narrow vibrational peaks followed by at least four broader maxima (Fig. 2). Several previous experimental studies ${ }^{12-16}$ lead to the conclusion that state $A^{2} \Sigma^{+}$is predissociated by a repulsive ${ }^{4} \Pi$ which correlates with the first dissociation asymptote (viz. , $\mathrm{Br}^{+}\left({ }^{3} P_{2}\right)+\mathrm{H}\left({ }^{2} S\right)$ at $\left.15.594 \mathrm{eV}\right)$. The crossing presumably takes place between the fourth and fifth vibrational levels of state $A^{2} \Sigma^{*}$. With the definitions given in Eq. (II. 2), the width of a broadened peak (FWHM) is equal to $2 \Gamma$ and the lifetime $\tau$ to $\hbar / 2 \Gamma$. A rough measurement led to ap- 
TABLE I. Energies (in eV) and intensities of the vibrational levels of state $A^{2} \Sigma^{+}$of $\mathrm{HBr}^{+}$. Intensities are determined by measuring the peak areas and not the relative heights. As a result of peak overlap, the values given for the predissociated levels $\left(v^{\prime}=4\right.$ to 8$)$ are not very accurate. It is, however, not crucial since these levels do not appear in the strong coupling limit formula.

\begin{tabular}{clc}
\hline \hline Vibrational level & Energies & Franck-Condon factors \\
\hline 0 & 15.288 & 0.09 \\
1 & 15.463 & 0.16 \\
2 & 15.607 & 0.19 \\
3 & 15.748 & 0.14 \\
4 & 15.89 & 0.11 \\
5 & 16.05 & 0.10 \\
6 & 16.17 & 0.08 \\
7 & 16.26 & 0.07 \\
8 & 16.34 & 0.06 \\
\hline
\end{tabular}

proximate values of $\Gamma \simeq 0.025 \mathrm{eV}$ and $\tau \simeq 1.5 \times 10^{-14} \mathrm{~s}$. The lifetime is thus estimated to be of the order of a half vibrational period. This value of $\tau$, when introduced into the Landau-Zener formula together with a reasonable estimate of the slopes leads to a value of the electronic coupling matrix element $V^{01}=\left\langle{ }^{4} \Pi\left|H^{80}\right|^{2} \Sigma^{*}\right\rangle$ equal to about $0.14 \mathrm{eV}$. The matrix element $V^{01}$ should not be confused with the $V$ of Eq. (II. 2) which is the total interaction matrix element, thus including the nuclear part. The value of $V^{\circ 1}$ is high, but not unreasonable. The probable electronic configurations at the crossing point ${ }^{14} \ldots(7 \sigma)^{2}(8 \sigma)^{1}$ $\times(4 \pi)^{4}$ for $A^{2} \Sigma^{+}$and $\ldots(7 \sigma)^{2}(8 \sigma)^{1}(4 \pi)^{3}(9 \sigma)^{1}$ for ${ }^{4} \Pi$ differ by a single spin orbital. In addition, the presence of a bromine atom brings about a large spin-orbit coupling constant $\left(\zeta_{2 \phi}=0.3 \mathrm{eV}\right.$ for the $\mathrm{Br}$ atom).

The corresponding correlation function is represented in Fig. 3. It is characterized by a rapid decrease followed by three recurrences whose maxima are all approximately equal to 0.5 . This suggests that the situation corresponds to the limit case described in Sec. III, i. e. , that the correlation function is given by $\sqrt{F_{1}(t)}$ with $F_{1}$ given by Eq. (III. 7). This implies that the wave

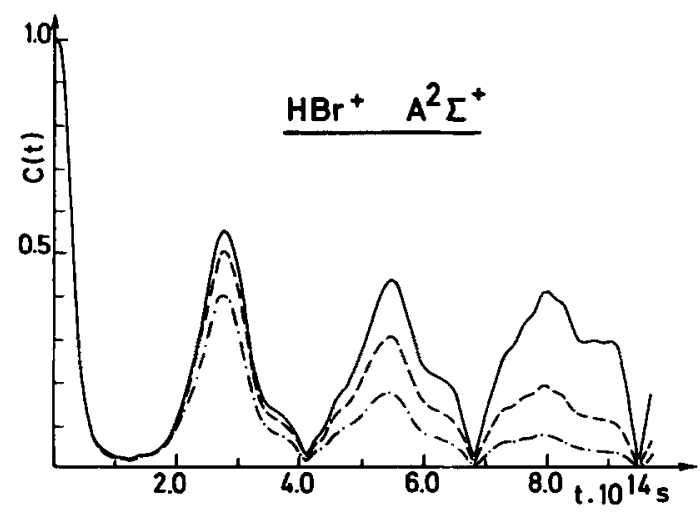

FIG. 3. Correlation function of the $A^{2} \Sigma^{+}$state of $\mathrm{HBr}^{+}$. Dotdash line: Fourier transform of the experimental photoelectron spectrum. Dashed line: same after correction for finite energy resolution. Solid line: same after correction for rotation.

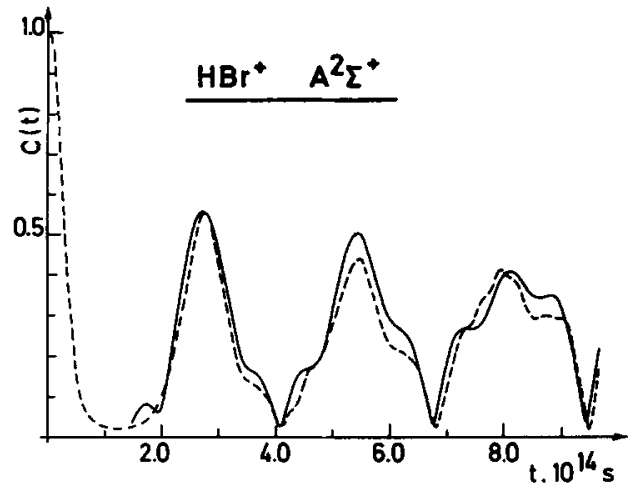

FIG. 4. Comparison between experimental and theoretical correlation functions of state $A^{2} \Sigma^{+}$of $\mathrm{HBr}^{+}$in the strong coupling limit. Dashed line: experimental function (the same as in Fig. 3). Solid line: calculated function [using the square root of $F_{1}$ given by Eq. (III. 7)].

packet splits into two parts after going across the nonadiabatic coupling zone, i. e., after a time equal to onehalf vibrational period. One part flies away to infinity, whereas the bound part, which remains trapped in the attractive potential is responsible for the recurrences of the correlation function. However, state $A^{2} \Sigma^{+}$is known to be strongly anharmonic $\left(\omega_{e}=1452 \mathrm{~cm}^{-1} ; \omega_{e} x_{e}\right.$ $\left.=52 \mathrm{~cm}^{-1}\right)$. As a result, very conspicuous flattening and deformation of the wave packet take place, as shown by the correlation function, whose maximum height at the recurrences slightly decreases while evidence of distortion progressively shows up.

This simple interpretation is correct, as shown by the excellent agreement between the experimental correlation function (Fig. 3) and the square root of $F_{1}$, as given by Eq. (III. 7) (Fig. 4). The energy levels and FranckCondon factors are reported in Table $I$.

\section{CONCLUSIONS}

The excellent agreement between the calculated and experimental results asserts the validity of the qualitative picture according to which the first four vibrational levels of state $A^{2} \Sigma^{+}$are not predissociated within the time scale of our experiment, whereas the upper levels are strongly coupled to the continuum with a very short lifetime of the order of one-half vibrational period. Numerical studies of Eq. (III. 6) with energy levels and Franck-Condon factors adjusted to the present case led to a value of $\Gamma$ at least equal to $0.027 \mathrm{eV}$, in excellent agreement with the approximate value $(0.025 \mathrm{eV})$ deduced in the previous section from the width of the broadened peaks.

This spectroscopic language is entirely equivalent to the dynamic picture of a wave packet splitting into two parts after having crossed the nonadiabatic coupling zone. However, the study of the correlation function reveals aspects which are absent, or at least not very obvious in the energy spectrum: the further evolution and deformation of the trapped part, which can be visualized in a very pictorial and appealing way.

The problem may be trivial for a diatomic molecule, 
but becomes much more interesting in a polyatomic molecule. In practice, it is not easy to decide whether a predissociation is purely electronic or purely vibrational. ${ }^{17}$ In actual life, the predissociation of a polyatomic molecule involves coupling processes where both kinds of degrees of freedom play a role. For a polyatomic molecule undergoing a nonadiabatic process, there is no such thing as a crossing point, but rather a seam, a line, a locus whose energy is a function of the nuclear coordinates. Thus, electronic predissociation is most efficient in particular nuclear geometries, i. e., when one or several particular normal modes are excited. ${ }^{18}$ The same idea can be put forward by saying that energy gaps are "modulated" by the excitation of totally symmetric modes $^{6,7,19}$ The correlation function method, which has been tested here in a simple case should be adequate for a study of more complicated coupling schemes. This will be attempted in a forthcoming paper.

\section{ACKNOWLEDGMENTS}

We are grateful to Dr. A. J. Lorquet for a preliminary determination and analysis of the correlation function of state $A^{2} \Sigma^{+}$of $\mathrm{HBr}^{+}$. B. L. is indebted to the IRSIA (Belgium) for a fellowship. This work has been financlally supported by the Belgian Government (Action de Recherches Concertées) and by the FRFC (research grant).

\section{APPENDIX}

In the Appendix, we derive the analytical expression of $C(t)$ by the two methods mentioned in Sec. III, in the case where two resonances $E_{\alpha}$ and $E_{\beta}$ are coupled to the continuum.

(A) When $|\Psi(t)\rangle$ is expanded in the set of eigenfunctions of $H_{0}, C(t)$ is given by Eq. (III. 3), and one has to solve the coupled equations (III. 2) with $N=n+1$.

It is convenient to make the following substitution ${ }^{20}$ :

$$
a_{\alpha, \beta}(t)=b_{\alpha, \beta}(t) \exp \left(-i E_{\alpha, \beta} t / \hbar\right) \text {. }
$$

Equations (III. 2) may then be written in matrix form:

$$
\dot{a}(t)=-i / \hbar a_{\mathrm{a}}(t),
$$

where $a(t)$ is the column vector

$$
\left(\begin{array}{l}
a_{\alpha}(t) \\
a_{B}(t)
\end{array}\right)
$$

and $\alpha$ is a symmetric, non-Hermitian constant matrix:

$$
\alpha=\left(\begin{array}{cc}
E_{\alpha}-i \Gamma & -i \Gamma \\
-i \Gamma & E_{\beta}-i \Gamma
\end{array}\right) \text {. }
$$

$a$ is diagonalized by a complex, or thogonal but not uni tary matrix $\delta$, so that

$$
\operatorname{sas}^{-1}=\Lambda \text {. }
$$

After multiplication by $\delta$, Eq. (A2) can be written:

$$
\delta \mathbf{a}(t)=-i / \hbar s a s^{-1} \mathbf{a}(t)
$$

from which one gets:

$$
\mathrm{sa}(t)=\exp (-i \Lambda t / \hbar) \mathrm{a}(0)
$$

or equivalently:

$$
a(t)=\mathfrak{M a}(0)=\mathfrak{M b}(0)
$$

with

$$
\text { গi }=\mathcal{S}^{-1} \exp (-i \Lambda t / \hbar) S .
$$

The eigenvalues of $a$ are

$$
\lambda_{1,2}=\left(E_{\alpha}+E_{\beta} \pm \Delta E \delta\right) / 2-i \Gamma
$$

with $\delta$ and $R$ defined, respectively, by Eqs. (III. 12) and (III. 11). We shall give details of calculations for $\delta$ real, i. e. , $2 \Gamma<\Delta E$ which corresponds to the most widely encountered situation. Nevertheless the case $2 \Gamma>\Delta E$ will be discussed in part $B$ of this Appendix. Starting from the eigenvalues (A6), the or thogonal nonunitary matrix $S$ is easily obtained:

$$
\delta=\left(\begin{array}{ll}
\alpha /\left(1+\alpha^{2}\right)^{1 / 2} & \beta /\left(1+\beta^{2}\right)^{1 / 2} \\
1 /\left(1+\alpha^{2}\right)^{1 / 2} & 1 /\left(1+\beta^{2}\right)^{1 / 2}
\end{array}\right)
$$

with $\alpha=-2 i R /(1+\delta)$ and $\beta=-2 i R /(1-\delta)$. From definition (A5), one gets after some algebra:

$$
\mathfrak{R}=\exp \left\{-\left[2 \Gamma+i\left(E_{\alpha}+E_{B}\right)\right] t / 2 \hbar\right\}\left(\begin{array}{cc}
\frac{\delta \cos \phi t+i \sin \phi t}{\delta} & -\frac{2 R}{\delta} \sin \phi t \\
-\frac{2 R}{\delta} \sin \phi t & \frac{\delta \cos \phi t-i \sin \phi t}{\delta}
\end{array}\right)
$$

or $\mathfrak{T}=\exp \left\{-\left[2 \Gamma+i\left(E_{\alpha}+E_{\beta}\right)\right] t / 2 \hbar\right\} \mathfrak{R}^{\prime}$ with $\phi$ given by Eq. (III. 13).

After substitution of (A8) into (A4) and (A1), one obtains $b_{\alpha}(t)$ and $b_{\beta}(t)$, so that Eq. (III. 3 ) becomes

$$
\begin{gathered}
C(t)=\mid \sum_{j}^{n-1} T_{j} \exp \left(-i E_{\jmath} t / \hbar\right)+\exp \left\{-\left[2 \Gamma+i\left(E_{\alpha}+E_{\beta}\right) t\right] / 2 \hbar\right\} \\
\quad\left[T_{\alpha} 9 \pi_{11}^{\prime}+T_{B} \mathfrak{g M}_{22}^{\prime}+2\left(T_{\alpha} T_{B}\right)^{1 / 2} \mathfrak{M}_{12}^{\prime}\right] \mid
\end{gathered}
$$

with $T_{j}=\left|b_{j}(0)\right|^{2}$, which then leads to Eq. (III. 6).

(B) An alternative way to derive $C(t)$ consists in expanding $|\Psi(t)\rangle$ in the set of eigenfunctions $\left|\Psi_{E}\right\rangle$ of $H_{T}$; $C(t)$ takes the form given by Eq. (III. 4).

One has then to calculate the symmetric matrix $I_{l k}(t)$ whose elements are defined by Eq. (III. 5). Let us remark that, at time $t=0$, this matrix is equal to the unity matrix, so that the following relation results: 


$$
\sum_{n} T_{0 n} I_{n k}(0)=T_{0 k}
$$

Mies and Krauss ${ }^{8}$ have derived the analytical expression of the $a_{n}(E)$ :

$$
a_{n}(E)=\frac{V_{n}(E)}{E-E_{n}} Z(E)
$$

with $Z(E)=[1+i A(E)]^{-1}$ and

$$
A(E)=\pi \sum_{j}^{N}\left|V_{j}(E)\right|^{2} /\left(E-E_{j}\right) .
$$

Taking account of the simplifying hypothesis of Eqs. (II. 2), $I_{l k}(t)$ becomes, after substitution by Eq. (A11),

$$
I_{l k}(t)=\frac{\Gamma}{\pi} \int d E \frac{\left(E-E_{t}\right)\left(E-E_{k}\right) \sum_{j \neq l_{1}}^{N}\left(E-E_{\jmath}\right)^{2} \exp (-i E t / \hbar)}{\sum_{j}^{2 N}\left(E-\lambda_{j}\right)},
$$

where $\lambda_{j}$ are the complex roots of the following $2 N$ degree polynomial $P(E)$ given by

$$
P(E)=\prod_{j}^{N}\left(E-E_{j}\right)^{2}+\Gamma^{2}\left[\sum_{j}^{N} \prod_{i \neq j}^{N}\left(E-E_{i}\right)\right]^{2},
$$

which is the same for all the $I_{l k}(t)$.

Each $I_{l k}(t)$ may then be evaluated by contour integration in the complex plane. The contour coincides with the infinite half-circle across the negative imaginary axis. The residues are therefore multiplied by $(-2 \pi i)$. The only difficulty of the method is the calculation of the complex roots $\lambda_{1}$ which requires a great numerical precision.

If there are only two resonances $E_{\alpha}$ and $E_{\beta}$, the four complex roots of $P(E)$ depend on the parameters $\delta$ and $R$ defined by Eqs. (III. 12) and (III.11). Two cases must be distinguished according to the real or imaginary character of $\delta$. In the real case corresponding to $2 \Gamma<\Delta E$, one gets:

$$
\begin{aligned}
& \lambda_{1,2}=\left(E_{\alpha}+E_{\beta} \pm \Delta E \delta\right) / 2-i \Gamma, \\
& \lambda_{3,4}=\left(E_{\alpha}+E_{\beta} \pm \Delta E \delta\right) / 2+i \Gamma,
\end{aligned}
$$

$\lambda_{1,2}$ only are located inside the integration contour. ${ }^{20}$ Evaluation of the two residues leads to:

$$
\begin{aligned}
& I_{\alpha \alpha}(t)=B(t)(\delta \cos \phi t+i \sin \phi t), \\
& I_{\beta B}(t)=B(t)(\delta \cos \phi t-i \sin \phi t), \\
& I_{\alpha \beta}(t)=-B(t)(2 \Gamma / \Delta E \delta) \sin \phi t,
\end{aligned}
$$

with $B(t)=\exp \left\{-\left[2 \Gamma+i\left(E_{\alpha}+E_{\beta}\right)\right] t / 2 \hbar\right\} / \delta$.
In the case where $\delta$ is imaginary, i. e., $2 \Gamma>\Delta E$, the same for $m$ is obtained but $\delta$ is replaced by a quantity $\gamma$ $=\left(4 R^{2}-1\right)^{1 / 2}$ and the cos and sin functions by cosh and sinh.

After substitution of Eqs. (A13) into Eq. (III. 4), the final expression (III. 9) is again recovered.

${ }^{1}$ E. J. Heller, J. Chem. Phys. 68, 2066, 3891 (1978); Acc. Chem. Res. 14, 368 (1981); in Potential Energy Surfaces and Dynamics Calculations, edited by D. G. Truhlar (Plenum, New York, 1981), p. 103. D. J. Tannor and E. J. Heller, J. Chem. Phys. 77, 202 (1982).

${ }^{2}$ A. J. Lorquet, J. C. Lorquet, J. Delwiche, and M. -J. HubinFranskin, J. Chem. Phys. 76, 4692 (1982) (part I of this series).

${ }^{3}$ B. Leyh, J. C. Lorquet, M. -J. Hubin-Franskin, and J. Delwiche (submitted).

${ }^{4}$ K. Freed, Topics Appl. Phys. 15, 23 (1976); P. Avouris, W. M. Gelbart, and M. A. El Sayed, Chem. Rev. 77, 793 (1977); R. M. Hochstrasser and Ch. Marzzacco, J. Chem. Phys. 49, 971 (1968).

${ }^{5} \mathrm{D}$. Dehareng, X. Chapuisat, J. C. Lorquet, C. Galloy, and R. Raseev, J. Chem. Phys. 78, 1246 (1983).

${ }^{6}$ H. Köppel, L. S. Cederbaum, and W. Domcke, J. Chem. Phys. 77, 2014 (1982).

${ }^{7}$ H. Köppel, Chem. Phys, 77, 359 (1983).

${ }^{8}$ F. H. Mies and M. Krauss, J. Chem. Phys. 45, 4455 (1966).

${ }^{9}$ U. Fano, Phys. Rev. 124, 1866 (1961).

${ }^{10} \mathrm{C}$. Cohen-Tannoudji, B. Diu, and F. Laloë, Mécanique Quantique (Hermann, Paris, 1977), Tome II, p. 1341.

${ }^{11} \mathrm{C}$. W. Gear, Numerical Initial Value Problems in Ordinary Differential Equations (Prentice-Hall, New York, 1971).

${ }^{12}$ M. J. Haugh and D. Bayes, J. Phys. Chem. 75, 1472 (1971).

${ }^{13}$ R. F. Barrow and A. D. Caunt, Proc. R. Soc. London Ser. A 66, 617 (1953).

${ }^{14} \mathrm{G}$. Mathieu, H. Wankenne, and J. Momigny, Chem. Phys. Lett. 17, 260 (1972).

${ }^{15} \mathrm{~J}$. Lebreton, J. Chim. Phys. 70, 1189 (1973).

${ }^{16} \mathrm{~J}$. Delwiche, P. Natalis, J. Momigny, and J. E. Collin, J. Electron. Spectrosc. Related Phenom. 1, 219 (1972).

${ }^{17} \mathrm{G}$. Herzberg, Molecular Spectra and Molecular Structure. III. Electronic Spectra and Electronic Structure of Polyatomic Molecules (Van Nostrand, Princeton, 1967), p. 458.

${ }^{18}$ A. J. Lorquet, J. C. Lorquet, and W. Forst, Chem. Phys. $51,253(1980)$.

${ }^{19}$ H. Köppel, W. Domcke, L. S. Cederbaum, and W. von Niessen, J. Chem. Phys. 69, 4252 (1978). L. S. Cederbaum, H. Köppel, and W. Domcke, Int. J. Quantum Chem. Symp. 15, 251 (1981).

${ }^{20}$ M. Bixon, J. Jortner, and Y. Dothan, Mol. Phys. 17, 109 (1969). 